\title{
Streptavidin Monolayer-Crystal Affinity Grids: A Step Toward Controlling What Happens During Cryo-EM Sample Preparation
}

\author{
Robert M. Glaeser ${ }^{1}$, Bong-Gyoon Han $^{1}$ and Jamie H. D. Cate ${ }^{1,2}$ \\ 1. Molecular Biophysics and Integrated Bioimaging Division, Lawrence Berkeley National Laboratory, \\ University of California, Berkeley, CA, USA \\ 2. Department of Molecular and Cell Biology, California Institute of Quantitative Biosciences, and \\ Department of Chemistry, University of California, Berkeley, CA, USA
}

When single-particle cryo-EM specimens look good, the standard picture shown schematically in Figure $1 \mathrm{~A}$ accounts well for what is observed. When, however, there are either too few or too many particles in the field of view, or particles adopt preferential orientations, or particles are damaged and/or aggregated, this picture must be false. In fact, even when particles look good, the standard picture may still be incorrect. What has largely been missing is the possibility that proteins may denature very rapidly at the air-water interface [1]. In fact, evidence for an alternative picture, like that indicated schematically in Figure 1B, was demonstrated experimentally for both ferritin and proteasome particles, in which native particles were shown to be adsorbed to a denatured-protein monolayer at the air-water interface [2]. With this background in mind, we have begun to investigate the binding of particles to streptavidin monolayer crystals, which can be used as a structure-friendly affinity support film [3]. Affinity grids in general have many desirable features; in the present context we focus on the fact that the immobilized particles cannot make contact with the air-water interface, at least as long as the thin film of surrounding buffer remains thicker than the particle itself.

We have developed a long shelf-life version of streptavidin (SA) affinity grids, which can be made well in advance, ready to be used whenever desired [3]. When backed by an optional, but preferred, thin carbon film, these support films are robust and tolerant of many sample-buffers, even SDS detergent, which would otherwise disrupt the monolayer crystals. The SA crystals themselves are well ordered, and thus the Fourier transforms of images provide a convenient indication of their resolution. Surprisingly, the resolution of the SA crystal also provides a convenient indication of the resolution of particles, such as the 70S E coli ribosomes shown in Figure 2A. To illustrate this, Figure 2B shows that a 2-D scatter plot of the resolution of the SA crystal vs the quality of the Thon rings consists mainly of two clusters, for both of which the resolution shown by the Thon rings is equally good. The resolution of the SA crystals, on the other hand is grouped either in the range $\sim 3 \AA$ to $4 \AA$ ("good images"), or in the range $\sim 5$ $\AA$ or worse ("bad images"). The 3-D reconstructions of ribosome particles boxed from the two types of images followed the same trend of being "good" or "bad", as is shown in Figure 3.

Although the use of affinity grids avoids denaturation of particles at the air-water interface when samples are placed initially on the grids (see Figure 1), the same problem still can occur if the film of remaining buffer becomes thinner than the diameter of the bound particles. We suggest that this may have been what happened for the images represented by the "circular" cluster of points in Figure 2 B, for which the resolution of both the SA crystal and the ribosome particles was poor. We thus believe that at least one further improvement is needed in a rational approach for making cryo-samples. Possibilities include placing an electron "transparent", hydrophilic "coverslip" - possibly a surfactant layer - on top of the buffer to prevent "dewetting" of the particles, or including larger particles to pin the air-water interface so as to prevent the layer of buffer from becoming thinner than a given, desired value [4]. 


\section{References:}

[1] RM Glaeser and BG Han, Biophysics Reports (2016) p.1.

[2] H Yoshimura, T Scheybani and W Baumeister, Langmuir 10 (1994), p. 3290.

[3] BG Han et al, J Struct Biol 195 (2016) p.238.

[4] The authors acknowledge funding from NIH grants P01 GM051487 and R01 GM065050. We thank Dr. Arto Pulk for providing the sample of randomly biotinylated 70S ribosome particles.
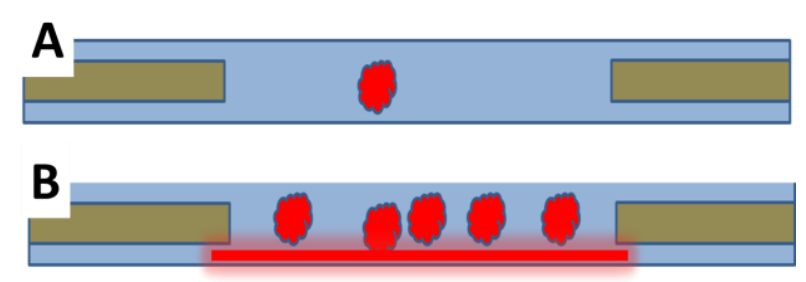

Figure 1. The standard picture of a cryo-EM specimen, (A), shows particles embedded in the middle of a thin slab of vitreous buffer. A second possibility, shown in (B), is that the first protein particles to hit the air-water interface rapidly become denatured, and additional particles then stick to the denatured-protein film before excess buffer is blotted away.
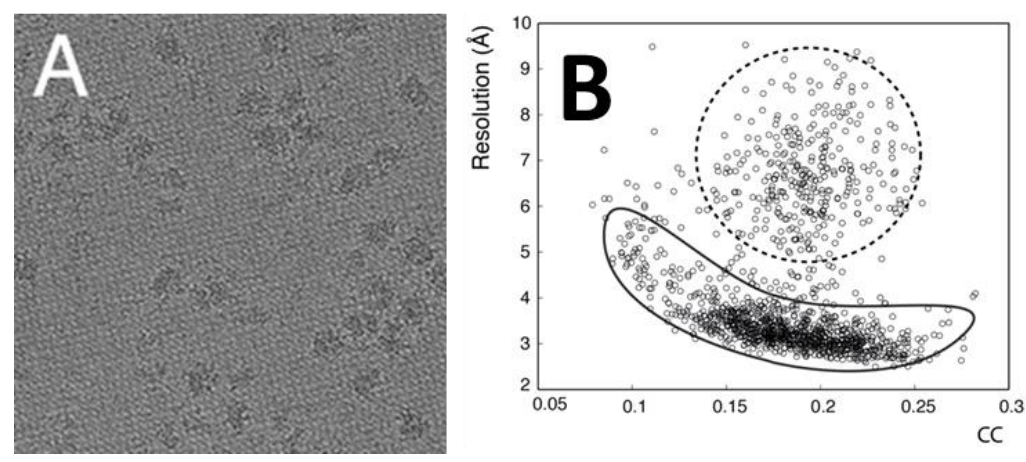

Figure 2. (A) Cryo-EM image of $E$ coli $70 \mathrm{~S}$ ribosome particles bound to a streptavidin monolayer-crystal support film. (B) Scatter plot showing the resolution of Bragg spots in the Fourier transforms of images, shown on the y-axis, and a measure of the goodness of Thon rings, shown on the $\mathrm{x}$-axis. Outlines highlight the clustering of data points.

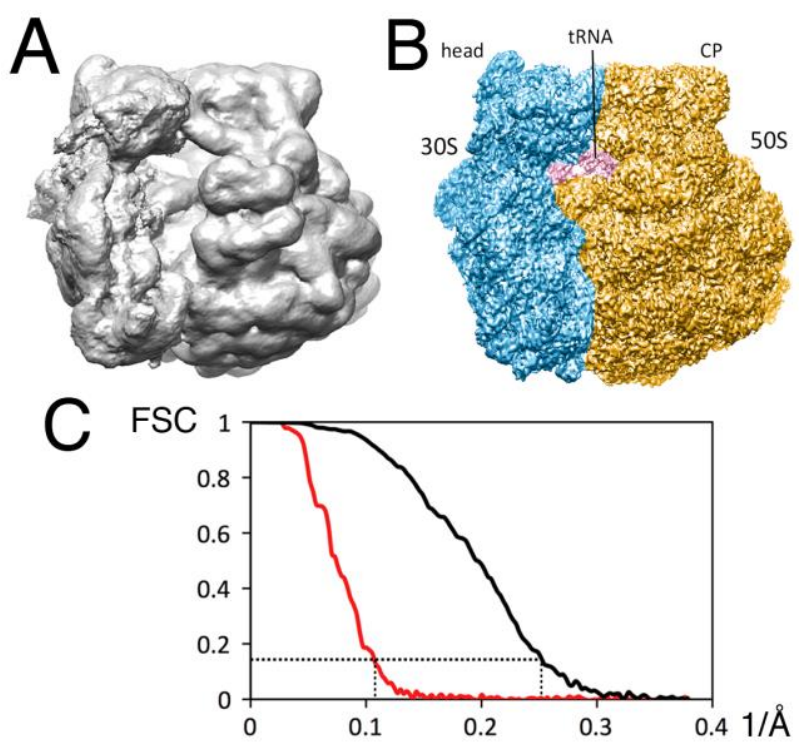

Figure 3. Comparison of 3-D reconstructions obtained from 22,697 E coli $70 \mathrm{~S}$ ribosomes boxed, in each case, from "bad" images and from "good" images, respectively. (A) The map obtained from images for which the resolution of the streptavidin crystal was $5 \AA$ or worse. (B) The map obtained from images for which the resolution of the streptavidin crystal was $4 \AA$ or better. (C) FSC curves, computed by the "gold standard" method. The resolution was only $9.5 \AA$ (red curve) for the map in (A), but it was about 4 $\AA$ (black curve) for the map in (B). 